Pacific Journal of Mathematic 


\title{
EIGENVALUES OF SEMINORMAL OPERATORS, EXAMPLES
}

\author{
R. W. Carey and J. D. Pincus
}

If $T^{*}$ is completely hyponormal and [T, $\left.T^{*}\right]$ has one dimensional range, a necessary and sufficient condition for a point $z$ to belong to the point spectrum of $T$ is known. Using this criterion two examples are constructed.

In the first example the point spectrum of $T$ is empty, in the second example the spectrum of $T$ is nowhere dense but almost every point of it is an eigenvalue.

The construction of both examples uses results about trigonometric series and the so-called principal function map $T \leftrightarrow g$ which associates with every bounded operator $T$ with $T T^{*}-T^{*} T \equiv 2 / \pi C$ trace class a Lebesgue summable function $g(\nu, \mu)$ defined on $\sigma(T)$, the spectrum of $T$.

The present paper will only consider the relatively simple case where $C$ has one dimensional range.

Let $T=U+i V$ be the Cartesian decomposition of $T$. With $C=k \otimes k$ it is known [4], [9] that

$$
\begin{aligned}
1 & +\frac{1}{\pi i}\left((V-l)^{-1}(U-z)^{-1} k, k\right) \\
& =\exp \left\{\frac{1}{2 \pi i} \iint g(\nu, \mu) \frac{d \nu}{\nu-l} \frac{d \mu}{\mu-z}\right\}
\end{aligned}
$$

for some function $g(\nu, \mu)$ with

$$
\begin{aligned}
& 0 \leqq g(\nu, \mu) \leqq 1 \quad \text { a.a. } \nu, \mu \\
& \iint g(\nu, \mu) d \nu d \mu=2 \text { trace } C .
\end{aligned}
$$

On the other hand, given any compactly supported measurable function satisfying (1) there exists an operator $\mathscr{T}$ which is completely hyponormal and for which $\mathscr{T}_{\mathscr{T}^{*}}-\mathscr{T}^{*} \mathscr{T} \equiv 2 / \pi \mathscr{C}$ is of one dimensional range such that

$$
\begin{aligned}
1 & +\frac{1}{\pi i}\left((\mathscr{V}-l)^{-1}(\mathscr{U}-z)^{-1} \mathscr{K}, \mathscr{K}\right) \\
& =\exp \left\{\frac{1}{2 \pi i} \iint g(\nu, \mu) \frac{d \nu}{\nu-l} \frac{d \mu}{\mu-z}\right\}
\end{aligned}
$$

where $\mathscr{T}=\mathscr{C}+i \mathscr{Y}$ is the cartesian decomposition of $\mathscr{T}$ and $\mathscr{C}=$ $\mathscr{K} \otimes \mathscr{K}$. 
For the proof of this result see [5]. We remark that it is also known that all such $\mathscr{T}$ 's are unitarily equivalent.

Let $\sigma_{p}(T)$ denote the point spectrum of $T$. We will use the following theorem, established in [6].

THeOREM 1. $z \in \sigma_{p}(T)$ if and only if, with $d A$ denoting area measure,

$$
\int_{B_{\varepsilon}} \int \frac{1-g(\zeta)}{|\zeta-z|^{2}} d A<\infty
$$

for some ball $B_{\varepsilon}$ centered at $z$, and $g(\zeta) \equiv g(\nu, \mu), \zeta=\mu+i \nu$.

Let $\chi_{F}$ denote the characteristic function of a set $F$. We will say throughout the following paragraphs that a set $F$ which is a subset of the real line has one dimensional density positive at $p$ if $\int_{p_{-\delta}}^{p+\tilde{o}} \chi_{F}(t) d t \neq 0$ for every $\delta>0$. A set $F$ which is a subset of the plane is said to have positive two dimensional density at $p$ provided that $\int_{|\gamma-p|<\delta} \int \chi_{F}(\gamma) d A \neq 0$ for every positive $\delta$.

EXAMPLE 1. An operator $T$ with $T^{*}$ hyponormal, $T T^{*}-T^{*} T$ of one dimensional range, and $\sigma_{p}(T)=\varnothing$.

We will exhibit a principal function $g(\zeta)$ with

$$
\int_{B \varepsilon} \int \frac{1-g(\zeta)}{|\zeta-z|^{2}} d A=\infty \text { for all } z
$$

The construction of Example 1. Let $F$ be a perfect, compact, nowhere dense set, lying in an interval $(a, b)$ with $a>0$, of positive Lebesgue measure and positive density at each of its points.

Let

$$
\Omega=\left\{q e^{i \varphi}: q \in F, 0 \leqq \varphi \leqq 2 \pi\right\} .
$$

It follows easily from this definition that $\Omega$ is compact, perfect, and nowhere dense.

Lemma 1. $\Omega$ has positive two dimensional density at each of its points.

Proof. If the proposition were false, we would have

$$
\int_{|\zeta-z|<\varepsilon} \int \chi_{\Omega}(\zeta) d A=0,
$$


with $\chi_{\Omega}$ the characteristic function of $\Omega$, for some $z$ in $\Omega$ and some positive $\varepsilon$.

But

$$
\int_{|\zeta-z|<\varepsilon} \int \chi_{\Omega}(\zeta) d A=\int_{|\eta|<\varepsilon} \int \chi_{\Omega}(\eta+z) d A .
$$

Let $\eta=\rho e^{i \theta}$, then

$$
\int_{|\eta|<\varepsilon} \int_{\Omega}(\eta+z) d A=\int_{0}^{2 \pi} d \theta \int_{0}^{\varepsilon} \chi_{\Omega}\left(\rho e^{i \theta}+z\right) \rho d \rho=0 .
$$

Thus,

$$
\int_{0}^{\varepsilon} \chi_{\Omega}\left(\rho e^{i \theta}+z\right) \rho d \rho=0 \quad \text { a.a. } \theta,
$$

and thus

$$
\int_{0}^{\varepsilon} \chi_{\Omega}\left(\rho e^{i \theta}+z\right) d \rho=0 \quad \text { a.a. } \theta \text {. }
$$

Also setting $\varphi=\theta-\pi$, we have

$$
\int_{-\pi}^{\pi} d \theta \int_{0}^{\varepsilon} \chi_{2}\left(\rho e^{i(\theta-\pi)}+z\right) \rho d \rho=0
$$

and hence

$$
\int_{0}^{\varepsilon} \chi_{\Omega}\left(\rho e^{i(\theta-\pi)}+z\right) d \rho=0 \quad \text { a.a. } \theta .
$$

Thus, for almost all $\theta$, both of the sets: $\left\{\rho: \rho \in[0, \varepsilon]\right.$ and $\rho e^{i \theta}+$ $z \in \Omega\}, \quad\left\{\rho: \rho \in[0, \varepsilon]\right.$ and $\left.\rho e^{i(\theta-\pi)}+z \in \Omega\right\}$ have zero one dimensional measure.

We will show that this contradicts the fact that $F$ has positive density at $r=|z|$.

The set of all $\rho$ in $(0, \varepsilon)$ with $\rho e^{i \theta}$ in $\Omega-z$ coincides with the set of all $\rho$ in $(0, \varepsilon)$ for which $\rho \in e^{i \theta}(\Omega-z)$.

Let $\Lambda_{(1)}=\left\{q: q>0\right.$ and $e^{-i \theta}\left(q e^{i \varphi}-z\right)$ is real for some $\left.\varphi\right\}$.

A point $q$ is in $\Lambda_{(1)}$ if and only if $q \sin (\varphi-\theta)-r \sin (\alpha-\theta)=0$ for some $\varphi$.

We may suppose that $0<|\sin (\alpha-\theta)|<1$.

We consider two cases:

Case 1.

$$
\cos (\alpha-\theta)>0 \text {. }
$$

For $|q-r|$ sufficiently small, there exists an angle $\varphi(q)$ such that $\cos (\varphi(q)-\theta)>0$ and $\sin (\varphi(q)-\theta)=r / q \sin (\alpha-\theta)$. Let 


$$
\begin{aligned}
F_{0}(q) & =\operatorname{Re}\left\{e^{i \theta}\left[q e^{-i \varphi(q)}-r e^{i \alpha}\right]\right\} \\
& =\left(q^{2}-r^{2} \sin ^{2}(\alpha-\theta)\right)^{1 / 2}+r \cos (\alpha-\theta) .
\end{aligned}
$$

Note that $F_{0}(r)=0$ and $F_{0}(q)$ is a strictly monotone increasing function of $q$ for $q$ larger than $r$.

Thus, for $q$ in a right hand neighborhood of $r$ the values of $F_{0}(q)$ range through some interval $(0, \delta)$.

This shows that $F$ has zero right hand density at $r$.

To discuss the left hand density of $F$ at $r$, we consider the angle $\theta-\pi$.

Note that $\cos (\alpha-\theta+\pi)<0$, and

$$
F_{\pi}(q) \equiv \operatorname{Re}\left(e^{-i(\theta-\pi)}\left[q e^{-i \varphi(q)}-r e^{i \alpha}\right]\right)=-F_{0}(q) .
$$

Thus, for $q$ less than $r$ and $|q-r|$ sufficiently small the range of $F_{\pi}(q)$ lies in $(0, \delta)$.

Thus $F$ has zero left hand density at $r$, and hence $F$ has zero density at $r$. This is a contradiction.

Case 2.

$$
\cos (\alpha-\theta)<0 \text {. }
$$

For $|q-r|$ sufficiently small there exists an angle $\varphi(q)$ such that $\cos (\varphi(q)-\theta)<0$ and $\sin (\varphi(q)-\theta)=r / q \sin (\alpha-\theta)$. With $F_{0}(q)$ as before we have

$$
F_{0}(q)=-\sqrt{q^{2}-r^{2} \sin ^{2}(\alpha-\theta)}-r \cos (\alpha-\theta) .
$$

Note that $F_{0}(r)=0$ and $F_{0}(q)$ is strictly increasing. Thus, for $q<r$ and $r-q$ sufficiently small the values of $F_{0}(q)$ belong to $(0, \delta)$.

This shows that $F$ has zero left hand density at $r$. Again,

$$
F_{\pi}(q)=\sqrt{q^{2}-r^{2} \sin ^{2}(\alpha-\theta)}+r \cos (\alpha-\theta)
$$

and $F_{\pi}(r)=0$. Since $F_{\pi}(q)$ is now increasing in $q$ there is an interval $(r, r+a)$ on which $F_{\pi}(q)$ ranges through $(0, \delta)$. Thus, $F$ has zero right hand density at $r$; hence, $F$ has zero density at $r$ which is a contradiction. The proof of the proposition is now complete.

So far $F$ has been any perfect compact nowhere dense set in $(a, b)$ with positive Lebesgue measure and positive density at each of its points.

Now we make use of a construction of N. N. Luzin [11] to choose the $F$ more carefully.

Luzin has provided us with an example of a measurable set $E$ contained in an interval $(a, b)$ with $a>0$ such that with $\delta$ any positive number 


$$
\int_{0}^{\delta}\left|\frac{\chi_{E}(p+t)-\chi_{E}(p-t)}{t}\right| d t=\infty
$$

for almost all $p$ in $(a, b)$, where $\chi_{E}$ is the characteristic function of $E$. Let the set of such $p$ be called $S$.

Choose, for the remainder of this paper, $F \subset S \cap E$. Note that

$$
\left|\chi_{E}(p+t)-\chi_{E}(p-t)\right| \leqq\left(1-\chi_{E}(p+t)\right)+\left(1-\chi_{E}(p-t)\right) \text {. }
$$

Hence,

$$
\begin{aligned}
& \int_{0}^{o} \frac{\left|\chi_{E}(p+t)-\chi_{E}(p-t)\right|}{t} d t \\
& \quad \leqq \int_{0}^{\delta} \frac{1-\chi_{E}(p+t)}{t} d t+\int_{0}^{o} \frac{1-\chi_{E}(p-t)}{t} d t .
\end{aligned}
$$

Since $F \subset E, 1-\chi_{E}(p \pm t) \leqq 1-\chi_{F}(p \pm t)$. Thus,

$$
\begin{aligned}
& \int_{0}^{\delta} \frac{1-\chi_{E}(p+t)}{t} d t+\int_{0}^{\delta} \frac{1-\chi_{E}(p-t)}{t} d t \\
& \quad \leqq \int_{0}^{\delta} \frac{1-\chi_{F}(p+t)}{t} d t+\int_{0}^{\delta} \frac{1-\chi_{F}(p-t)}{t} d t=\infty
\end{aligned}
$$

for all $p \in F$, and hence for all real $p$.

We now define $g(\zeta)=\chi_{\Omega}(\zeta)$, the characteristic function of $\Omega$.

This function will be our choice of principal function.

By the result in [5] referred to above, there exists a completely nonnormal seminormal operator $T$ with one dimensional self-commutator and principal function $g(\zeta)$.

It is this operator $T$, for which we will later exhibit a concrete representation, which we claim has no point spectrum.

To show this it suffices by Theorem 1 to prove that

$$
\int_{B_{\varepsilon}} \int \frac{1-g(\zeta)}{|\zeta-z|^{2}} d A=\infty
$$

for all $z \in \Omega$.

We proceed with this demonstration.

There exist numbers $r_{1}$ and $r_{2}$ such that $0<r_{1}<r<r_{2}$, and a positive number $b$ such that

$$
\begin{aligned}
\int_{|\zeta-z|<\varepsilon} & \int \frac{1-g(\zeta)}{|\zeta-z|^{2}} d A \geqq \int_{r_{1}}^{r_{2}} \int_{\alpha-b}^{\alpha+b} \frac{1-g\left(\rho e^{i \theta}\right)}{\left|\rho e^{i \theta}-r e^{i \alpha}\right|^{2}} \rho d \rho d \theta \\
= & \int_{r_{1}}^{r_{2}} \int_{\alpha-b}^{\alpha+b} \frac{1-\chi_{F}(\rho)}{\left|\rho e^{i \theta}-r e^{i \alpha}\right|^{2}} \rho d \rho d \theta \\
= & \int_{r_{1}}^{r} \frac{\left[1-\chi_{F}(\rho)\right]}{r^{2}\left(1-\rho^{2} / r^{2}\right)}\left(\rho \int_{\alpha-b}^{\alpha+b} \frac{\left(1-\rho^{2} / r^{2}\right) d \theta}{1+(\rho / r)^{2}-2 \rho / r \cos (\theta-\alpha)}\right) d \rho \\
& +\int_{r}^{r_{2}} \frac{\left[1-\chi_{F}(\rho)\right]}{\rho^{2}\left(1-r^{2} / \rho^{2}\right)}\left(\rho \int_{\alpha-b}^{\alpha+b} \frac{\left(1-r^{2} / \rho^{2}\right) d \theta}{1+(r / \rho)^{2}-2 r / \rho \cos (\theta-\alpha)}\right) d \rho .
\end{aligned}
$$


Since

$$
\rho \int_{\alpha-b}^{\alpha+b} \frac{\left(1-\rho^{2} / r^{2}\right)}{1+(\rho / r)^{2}-2 \rho / r \cos (\theta-\alpha)} d \theta
$$

and

$$
\rho \int_{\alpha-b}^{\alpha+b} \frac{\left(1-r^{2} / \rho^{2}\right) d \theta}{1+(r / \rho)^{2}-2 r / \rho \cos (\theta-\alpha)}
$$

are continuous functions of $\rho$ on $\left(r_{1}, r\right)$ and $\left(r, r_{2}\right)$ respectively; and since, on these intervals, these functions do not vanish we can conclude that the integrals are bounded below by positive constants $c_{1}$ and $c_{2}$.

Thus,

$$
\begin{aligned}
& \int_{|\zeta-z|<\varepsilon} \int \frac{1-g(\zeta)}{|\zeta-z|^{2}} d A \geqq c_{1} \int_{r_{1}}^{r} \frac{1-\chi_{F}(\rho)}{(r-\rho)(r+\rho)} d \rho \\
& \quad+c_{2} \int_{r}^{r_{2}} \frac{1-\chi_{F}(\rho)}{(\rho-r)(\rho+r)} d \rho \\
& \geqq \frac{c_{1}}{2 r} \int_{r_{1}}^{r} \frac{1-\chi_{F}(\rho)}{r-\rho} d \rho+\frac{c_{2}}{2 r_{2}} \int_{r}^{r_{2}} \frac{1-\chi_{F}(\rho)}{\rho-r} d \rho .
\end{aligned}
$$

However, we have already seen that

$$
\int_{0}^{\delta} \frac{1-\chi_{F}(p+t)}{t} d t+\int_{0}^{\delta} \frac{1-\chi_{F}(p-t)}{t} d t=\infty
$$

for all real $p$.

Hence,

$$
\int_{|\zeta-z|<\varepsilon} \frac{1-g(\zeta)}{|\zeta-z|^{2}} d A=\infty
$$

for all $z \in \Omega$.

For the purpose of gaining additional insight into the structure of $T$ we will exhibit a concrete representation for $T$ which is different from the singular integral representation furnished in [5].

To this end we will construct a new operator $\mathscr{T}$ defined on a doubly infinite direct sum space, having the form of a bilateral shift with operator weights, and which has $g(\zeta)$ as its principal function.

It will then follow that $T$ and $\mathscr{T}$ are unitarily equivalent.

Consider the polar decomposition of $T, T=W Q$. Now $0 \notin \sigma(T)$ because, by a theorem of [10], the essential support of the principal function is $\sigma(T)$. Thus, $W$ is unitary.

Let $D \equiv T T^{*}-T^{*} T$, and let $D=1 / \pi d \otimes d$. Then $W Q^{2} W^{*}-$ $Q^{2}=D$, and $W Q^{2}=\left(Q^{2}+D\right) W$. 
For $|z| \neq 1, \operatorname{Im} l \neq 0$, we can form the so-called polar determining function of $T$ :

$$
\varphi(l, z)=1+\frac{1}{\pi}\left(W(W-z)^{-1}\left(Q^{2}-l\right)^{-1} d, d\right) .
$$

By known results [4], there exists a function $g^{p}(\lambda, \tau)$ such that

$$
\varphi(l, z)=\exp \left\{\frac{1}{2 \pi i} \int_{R} \int_{C} g^{p}(\lambda, \tau) \frac{d \lambda}{\lambda-l} \frac{d \tau}{\tau-z}\right\} .
$$

A fundamental identity [6] asserts that

$$
g^{p}\left(\lambda^{2}, \tau\right)=g(\lambda \tau)
$$

for

$$
\zeta=\lambda \tau, \lambda>0 \text { and }|\tau|=1
$$

Furthermore,

$$
\varphi(l, 0)=\exp \left\{\int \frac{\zeta(\lambda)}{\lambda-l} d \lambda\right\}
$$

where

$$
\zeta(\lambda)=\frac{1}{2 \pi} \int_{0}^{2 \pi} g^{p}\left(\lambda, e^{i \theta}\right) d \theta=\frac{1}{2 \pi} \int_{0}^{2 \pi} g\left(\sqrt{\lambda} e^{i \theta}\right) d \theta .
$$

A well-known theorem asserts that there exists a positive measure $d \nu(\lambda)$ such that

$$
\exp \left\{\int \frac{\zeta(\lambda)}{\lambda-l} d \lambda\right\}=1+\frac{1}{\pi} \int \frac{d \nu(\lambda)}{\lambda-l} .
$$

Furthermore, since $\zeta(\lambda)$ is a characteristic function, $d \nu(\cdot)$ is a purely singular measure [1], [3].

Let $\mathscr{H}=L^{2}(d \nu)$, and set

$$
\mathscr{K}=\cdots \oplus \mathscr{H} \oplus \mathscr{H} \oplus \mathscr{H} \oplus \mathscr{H} \oplus \mathscr{H} \oplus \cdots .
$$

Let $\mathscr{W}$ be the bilateral shift of multiplicity equal to dimension $\mathscr{H}$ (i.e., infinity).

Define $V$ on $\mathscr{H}$ by setting

$$
V f(t)=t f(t), \quad f(t) \in L^{2}(d \nu) .
$$

Let $\chi(t)=1$ a.a. $t$ in support of $d \nu$, and define

$$
C=\frac{1}{\pi} \chi \otimes \chi \text { as an operator on } L^{2}(d \nu)
$$


and set $\mathscr{C}=\cdots \oplus 0 \oplus 0 \oplus C \oplus 0 \oplus 0 \oplus \cdots$ on $\mathscr{K}$. With $\mathscr{V}$ on $\mathscr{K}$ defined as $\mathscr{V}=\cdots \oplus V+C \oplus V+C \oplus V \oplus V \oplus V \oplus \cdots$ we have

$$
\mathscr{W} \mathscr{Y}=(\mathscr{Y}+\mathscr{C}) \mathscr{W}^{-} \text {. }
$$

But, let us define

$$
\Phi(l, z)=1+\frac{1}{\pi}\left(\mathscr{W}(\mathscr{W}-z)^{-1}(\mathscr{Y}-l)^{-1} \chi, \chi\right) .
$$

Then

$$
\Phi(l, z)=\exp \left\{\frac{1}{2 \pi i} \int_{R C} \int \frac{\tilde{g}(\lambda, \tau)}{(\lambda-l)(\tau-z)} d \lambda d \tau\right\}
$$

for some summable function $\widetilde{g}(\lambda, \tau)$ with $0 \leqq \widetilde{g}(\lambda, \tau) \leqq 1$.

Furthermore,

$$
\Phi(l, 0)=\exp \int \frac{\zeta(\lambda)}{\lambda-l} d \lambda
$$

Now

$$
\zeta(\lambda)=\frac{1}{2 \pi} \int_{0}^{2 \pi} \widetilde{g}\left(\lambda, e^{i \theta}\right) d \theta,
$$

thus, since $\zeta(\lambda)$ is the characteristic function of $F^{2}=\left\{\lambda^{2}, \lambda \in F\right\}$, we can conclude that $\widetilde{g}^{p}\left(\sqrt{\lambda}, e^{i \theta}\right)=\zeta(\lambda)$ a.a. $\theta$.

But the same reasoning applied to the equation

$$
\zeta(\lambda)=\frac{1}{2 \pi} \int_{0}^{2 \pi} g^{p}\left(\lambda, e^{i \theta}\right) d \theta
$$

tells us that $\zeta(\lambda)=g^{p}\left(\lambda, e^{i \theta}\right)$ a.a. $\theta$.

Hence $g^{p}\left(\lambda, e^{i \theta}\right)=\widetilde{g}^{p}\left(\lambda, e^{i \theta}\right)$, and thus $\mathscr{T} \equiv \mathscr{W}^{-} \mathscr{V}^{1 / 2}$ is unitarily equivalent to $T$.

We remark that representations of this type play an important role in the study of intertwining contractions. See the forthcoming study in [8]. As our only use of this representation here we mention that it is now quite easy to exhibit proper invariant subspaces for $T$ (hence $T^{*}$ ). For instance, if $\mathscr{F}=\left\{\Delta_{n}\right\}_{n=-1}^{-\infty}$ is an increasing family of Borel sets in $(-\infty, \infty)$ for which $E\left(\Delta_{n}\right) \neq 1$ for some $n$ (here $V+D=\int \lambda d E_{\lambda}$ is the spectral resolution for $V+D$ on the space $\left.L^{2}(d \nu)\right)$ and $\mathscr{H}_{n}=E\left(\Delta_{n}\right) \mathscr{H}$, then the closed subspace

$$
\mathscr{K}_{\mathscr{S}}=\cdots \oplus \mathscr{H}_{-2} \oplus \mathscr{H}_{-1} \oplus \mathscr{H} \oplus \mathscr{H} \oplus \cdots
$$

is different from $\{0\}$ and $\mathscr{K}$ and invariant for $\mathscr{T}$. 
EXAMPLE II. In contrast to the previous example we next exhibit a seminormal operator $T$ with $\sigma(T)$ nowhere dense such that Area $\left(\sigma(T) / \sigma_{p}(T)\right)=0$. In order to accomplish this it suffices to construct a set $K$ having positive planar density at each of its points such that the integral

$$
\iint \frac{1-\chi_{K}(\zeta)}{|\zeta-z|^{2}} d A
$$

is finite for almost all $z$ in $K$. To construct such a $K$ we rely on a subtle result of G. P. Tolstov in a version due to N. Bary (see page 466 of [2]), which we now state:

LEMma 2. Let $f$ be any measurable function definable in some interval $0 \leqq h \leqq h_{0}, f$ positive, monotonic, $\lim _{h \rightarrow 0} f(h)=0$. Let $[a, b]$ be any interval. If $0<\mu<b-a$, then there exists a perfect, nowhere dense set $F$, with $m(F)=\mu$, such that for $m$-almost all $x \in F$, there exists a positive number $\delta_{x}$ such that

$$
\frac{m[R \backslash F \cap(x, x+h)]}{2|h|}<f(|h|)
$$

for $0<|h|<\delta_{x}$.

For our purposes let $f(h)=h^{1+\alpha}$ where $\alpha$ is chosen to be positive. Let $F$ be a set with the properties stated in the above lemma corresponding to $h^{1+\alpha}$. By removing the set $G$ of points $x$ in $F$ where $F$ has zero density we obtain a closed set $F / G$, having positive density at each of its points and satisfying the conditions of lemma two. Since for the present purpose we need only the fact that such a set exists, we shall assume from this point on that $G$ is empty. Set $K=\{x+i y: x \in F$ and $2 \leqq y \leqq 3\}$. Since $F$ has positive linear density at each of its points it follows easily that $K$ is compact, nowhere dense and has positive planar density for all $z \in K$. Now, with $g(\cdot)$ equal to the characteristic function of $K$, let $T$ denote the corresponding seminormal operator [5]. It remains to show that the integral in (3) is finite for almost all $z$ in $K$. Let $\Omega$ denote the subset of $F$ for which the relation (4) is valid. Since $m(F \backslash \Omega)=0$, clearly Area $(K \backslash\{x+i y: x \in \Omega\})=0$. We shall now show that the integral in (3) is finite whenever $z=x+i y, x \in \Omega$ and $2 \leqq y \leqq 3$. To do this, it suffices to show that there exists a positive number $\delta$ for which the integral

$$
\int_{x-\delta}^{x+\delta} \int_{y-\delta}^{y+\delta} \frac{1-g(a+i b)}{(a-x)^{2}+(y-b)^{2}} d a d b
$$


is finite. Let $\delta=\delta_{x}$ be chosen as in the lemma for $f(h)=h^{1+\alpha}$. With $h=a-x, k=b-y$, the integral becomes

$$
\begin{aligned}
& \int_{\delta-}^{\delta} \int_{-\delta}^{\delta} \frac{1-g(h+x+i(k+y))}{h^{2}+k^{2}} d h d k \\
& \quad \leqq \int_{-\delta}^{\delta} \frac{1-\chi_{F}(h+x)}{h^{2}} d h \cdot\left\{\int_{-\delta}^{\delta} \frac{d k}{1+(k / h)^{2}}\right\} \\
& \quad=\int_{-\delta}^{\delta} \frac{1-\chi_{F}(x+h)}{|h|} \cdot 2 \arctan \left(\frac{\delta}{|h|}\right) d h \\
& \quad \leqq 2 \pi \int_{-\delta}^{\delta} \frac{1-\chi_{F}(x+h)}{|h|} d h .
\end{aligned}
$$

Now, this last integral is finite if and only if the series

$$
\sum_{n=1}^{\infty}\left\{\int_{-\delta / n}^{-\delta / n+1} \frac{1-\chi_{F}(x+h)}{|h|} d h+\int_{\delta / n+1}^{\delta / n} \frac{1-\chi_{F}(x+h)}{|h|} d h\right\} .
$$

is convergent. But

$$
\begin{aligned}
& \int_{-\delta / n}^{-\delta / n+1} \frac{1-\chi_{F}(x+h)}{|h|} d h \\
& \quad \leqq\left(\frac{n+1}{\delta}\right) m(R \backslash F \cap(x-\delta / n, x-\delta / n+1)) \\
& \quad \leqq\left(\frac{n+1}{\delta}\right) m(R \backslash F \cap(x-\delta / n, x)) \\
& \quad \leqq 2\left(\frac{n+1}{\delta}\right) \cdot\left(\frac{\delta}{n}\right) \cdot f\left(\frac{\delta}{n}\right)=2\left(\frac{n+1}{n}\right) \frac{\delta^{1+\alpha}}{n^{1+\alpha}} .
\end{aligned}
$$

Similarly, we can show that

$$
\int_{\delta / n+1}^{\delta / n} \frac{1-\chi_{F}(x+h)}{|h|} d h \leqq 2\left(\frac{n+1}{n}\right) \frac{\delta^{1+\alpha}}{n^{1+\alpha}} .
$$

But, $\sum_{n=1}^{\infty} 1 / n^{1+\alpha}<\infty$ since $\alpha>0$; therefore, the integral is finite and $x+i y$ is an eigenvalue for $T$.

REMARK. The operators considered above have the property that their spectrum is nowhere dense, and therefore coincides with the essential spectrum. In particular, these operators are quasitriangular in contrast to such operators as the unilateral shift. On the other hand, it is possible simply by choosing the principal function $g(\cdot)$ bounded away from 1 , to obtain operators $T$ with the unit disc for its spectrum yet $T$ has no eigenvalues. 


\section{REFERENCES}

1. N. Aronszajn and W. F. Donoghue, Jr., On exponential representations of analytic functions in the upper half plane with positive imaginary parts, J. D'Analyse Math., (1956-1957), 321-388.

2. N. Bary, A Treatise on Trigonometric Series, Pergamon Press, 1964.

3. R. W. Carey and W. D. Pepe, The phase shift and singular measures, Indiana U. Math. J., (1973), 1049-1066.

4. R. W. Carey and J. D. Pincus, An exponential formula for determining functions, Indiana Univ. Math. J., 23 (1974), 1031-1042.

5 . - Construction of seminormal operators with prescribed mosaic, Indiana Univ. Math. J., 23 (1979), 1155-1165.

6. On an invariant for certain operator algebras, preprint, to appear in Proc. Nat. Acad. Sci., (71) (1974), 1952-1956.

7. — The structure of intertwining isometries, Indiana U. Math. J., 22 (1973), 679-703.

8. - Intertwining Isometries $I I$, in preparation.

9. J. D. Pincus, Commutators and systems of singular integral equations, Acta Math. 121 (1968), 219-249.

10. The spectrum of semi-normal operators, Proc. Nat. Acad. of Sci., 68 (1971), 1684-1685.

11. N. N. Lusin, The integral and trigonometric series, 2nd. ed. Gostekhizdet, Moscow, 1951.

12. G. P. Tolstov, On points of density of linear measurable sets, Math. Sbornik, 10 (52) (1942), 249-264.

Received August 7, 1973. This work was partially supported by the National Science Foundation.

UNIVERSITY OF KENTUCKY

AND

State University of New York, Stony Brook 



\section{PACIFIC JOURNAL OF MATHEMATICS}

EDITORS

RICHARD ARens (Managing Editor)

University of California

Los Angeles, California 90024

\section{R. A. Beaumont}

University of Washington

Seattle. Washington 98105

\section{J. DugundjI}

Department of Mathematics University of Southern California Los Angeles, California 90007

D. Gilbarg and J. Milgram

Stanford University

Stanford. California 94305

\section{ASSOCIATE EDITORS}
E. F. BECKENBACH
B. H. NEUMANN
F. WOLF
K. Yoshida

\section{SUPPORTING INSTITUTIONS}

\author{
UNIVERSITY OF BRITISH COLUMBIA \\ CALIFORNIA INSTITUTE OF TECHNOLOGY \\ UNIVERSITY OF CALIFORNIA \\ MONTANA STATE UNIVERSITY \\ UNIVERSITY OF NEVADA \\ NEW MEXICO STATE UNIVERSITY \\ OREGON STATE UNIVERSITY \\ UNIVERSITY OF OREGON \\ OSAKA UNIVERSITY
}

\author{
UNIVERSITY OF SOUTHERN CALIFORNIA \\ STANFORD UNIVERSITY \\ UNIVERSITY OF TOKYO \\ UNIVERSITY OF UTAH \\ WASHINGTON STATE UNIVERSITY \\ UNIVERSITY OF WASHINGTON

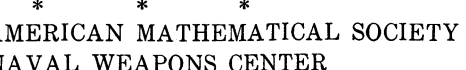

The Supporting Institutions listed above contribute to the cost of publication of this Journal, but they are not owners or publishers and have no responsibility for its content or policies.

Mathematical papers intended for publication in the Pacific Journal of Mathematics should be in typed form or offset-reproduced, (not dittoed), double spaced with large margins. Underline Greek letters in red, German in green, and script in blue. The first paragraph or two must be capable of being used separately as a synopsis of the entire paper. Items of the bibliography should not be cited there unless absolutely necessary, in which case they must be identified by author and Journal, rather than by item number. Manuscripts, in duplicate if possible, may be sent to any one of the four editors. Please classify according to the scheme of Math. Rev. Index to Vol. 39. All other communications to the editors should be addressed to the managing editor, or Elaine Barth, University of California, Los Angeles, California, 90024.

100 reprints are provided free for each article, only if page charges have been substantially paid Additional copies may be obtained at cost in multiples of 50 .

The Pacific of Journal Mathematics is issued monthly as of January 1966. Regular subscription rate: $\$ 72.00$ a year (6 Vols., 12 issues). Special rate: $\$ 36.00$ a year to individual members of supporting institutions.

Subscriptions, orders for back numbers, and changes of address should be sent to Pacific Journal of Mathematics, 103 Highland Boulevard, Berkeley, California, 94708.

\section{PUBLISHED BY PACIFIC JOURNAL OF MATHEMATICS, A NON-PROFIT CORPORATION}

Printed at Kokusai Bunken Insatsusha (International Academic Printing Co., Ltd.), 270, 3-chome Totsuka-cho. Shinjuku-ku, Tokyo 160. Japan.

Copyright (C) 1973 by Pacific Journal of Mathematics Manufactured and first issued in Japan 


\section{Pacific Journal of Mathematics}

\section{Vol. 52, No. $2 \quad$ February, 1974}

Harm Bart, Spectral properties of locally holomorphic vector-valued functions .....

J. Adrian (John) Bondy and Robert Louis Hemminger, Reconstructing infinite

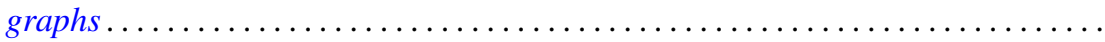

Bryan Edmund Cain and Richard J. Tondra, Biholomorphic approximation of planar

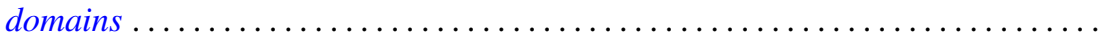

Richard Carey and Joel David Pincus, Eigenvalues of seminormal operators,

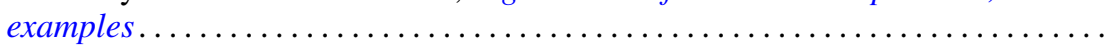

Tyrone Duncan, Absolute continuity for abstract Wiener spaces . . . . . . . . . . . . Joe Wayne Fisher and Louis Halle Rowen, An embedding of semiprime

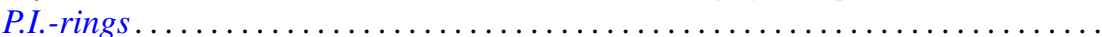

Andrew S. Geue, Precompact and collectively semi-precompact sets of semi-precompact continuous linear operators. . . . . . . . . . . . . . . .

Charles Lemuel Hagopian, Locally homeomorphic $\lambda$ connected plane continua ..... . Darald Joe Hartfiel, A study of convex sets of stochastic matrices induced by

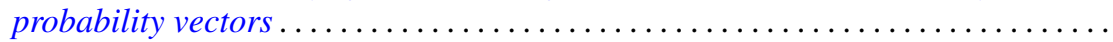

Yasunori Ishibashi, Some remarks on high order derivations $\ldots \ldots \ldots \ldots \ldots \ldots \ldots$ Donald Gordon James, Orthogonal groups of dyadic unimodular quadratic forms.

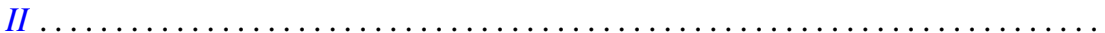

Geoffrey Thomas Jones, Projective pseudo-complemented semilattices . . . . . . . . . Darrell Conley Kent, Kelly Denis McKennon, G. Richardson and M. Schroder,

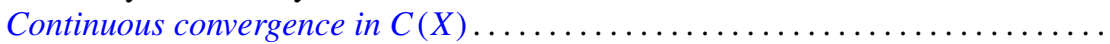

J. J. Koliha, Some convergence theorems in Banach algebras ...

Tsang Hai Kuo, Projections in the spaces of bounded linear oper

George Berry Leeman, Jr., A local estimate for typically real functions . .

475

Andrew Guy Markoe, A characterization of normal analytic spaces by the

homological codimension of the structure sheaf .........

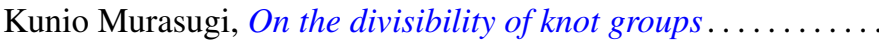

John Phillips, Perturbations of type I von Neumann algebras.

Billy E. Rhoades, Commutants of some quasi-Hausdorff matrices . .

David W. Roeder, Category theory applied to Pontryagin duality

Maxwell Alexander Rosenlicht, The nonminimality of the differential closure .

Peter Michael Rosenthal, On an inversion theorem for the general Mehler-Fock transform pair.

Alan Saleski, Stopping times for Bernoulli automorphisms

John Herman Scheuneman, Fundamental groups of compact complete locally affine complex surfaces. II. ........................

Vashishtha Narayan Singh, Reproducing kernels and operators with a cyclic vector. I. .

Peggy Strait, On the maximum and minimum of partial sums of random variables.

J. L. Brenner, Maximal ideals in the near ring of polynomials modulo 2 .

Ernst Gabor Straus, Remark on the preceding paper: "Ideals in near rings of polynomials over a field" ..........................

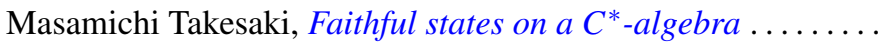

R. Michael Tanner, Some content maximizing properties of the regular simplex.

Andrew Bao-hwa Wang, An analogue of the Paley-Wiener theorem for certain

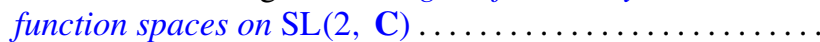

